\title{
Vorbeugendes Gewichtsmanagement - ein Leitfaden für die Praxis
}

\author{
Britta Dobenecker
}

Aktuelle Prävalenzuntersuchungen lassen vermuten, dass derzeit über $40 \%$ aller Hunde und Katzen adipös sind. Fettleibigkeit ist jedoch mehr als eine fütterungsbedingte Erkrankung, es handelt sich um ein komplexes Krankheitsbild. Tierärzte sind gefordert, sich an einer Strategie zu beteiligen, ein weiteres Ausufern dieses Trends zu verhindern. Aufklärung und konkrete Tipps an die Tierhalter spielen dabei eine zentrale Rolle. In einem aktuell erschienenen Kompendium wurden die wesentlichen Eckdaten dafür zusammengefasst. Das Handbuch „Vorbeugendes Gewichtsmanagement“ gibt Tierärzten wissenschaftlich fundierte Informationen, hilfreiche Tipps und praktische Materialien für die konkrete Beratung an die Hand. Im Folgenden lesen Sie ausgewählte Auszüge daraus.

\section{Aufgaben, Möglichkeiten und Grenzen des Tierarztes}

Bei der Prävention der Adipositas geht es darum, eine verantwortungsvolle Heimtierhaltung, eine bedarfsgerechte Fütterung und die Sicherstellung eines aktiven Lebensstils zu fördern. Der Tierarzt hat als kompetenter Ratgeber die besten Chancen, dies positiv zu beeinflussen. So kann er den Tierhalter dabei unterstützen, eine bedarfsdeckende und ausgewogene Fütterung zu praktizieren. Neben konkreten Fütterungsempfehlungen kann eine regelmäßige Gewichtskontrolle, u.a. bei jedem Tierarztbesuch, helfen, das Gewicht eines Tieres im Auge zu behalten und bei drohendem Übergewicht rechtzeitig gegenzulenken - ohne Schuldzuweisung, vielmehr durch praktische Hilfestellung und Tipps, die die individuelle Situation von Tier und Halter berücksichtigen.

Sensibel sollte dem Tierhalter vermittelt werden, dass der größte Fürsorgebeweis für sein Tier ist, es möglichst langfristig gesund, also auch schlank zu halten. Es gilt, gemeinsam zu überlegen, was getan werden kann, um dem Tier dauerhaft zum Idealgewicht und einem aktiven, gesunden Leben zu verhelfen.

\section{Dem Tierhalter Status und Ziel "begreiflich" machen}

Katze und Hund gelten als übergewichtig, wenn ihr Körpergewicht 15\% über dem Idealgewicht liegt, und als fettleibig, wenn es mehr als 30\% darüber liegt. Tierhalter können mit diesen Zahlen oft wenig anfangen. Sie brauchen es konkreter, sichtbar, fühlbar und messbar.

Im ersten Schritt sollte der aktuelle Ernährungszustand des Tieres ermittelt werden. Dazu gehört selbstverständlich das Wiegen. Für den Halter weitaus anschaulicher sind aber die visuelle Beurteilung sowie die haptische Einschätzung. Ein gutes Instrument hierfür ist der WALTHAM $^{\circledR}$ S.H.A.P.E ${ }^{\mathrm{TM}}$ Guide ( ${ }^{\text {Abb. } 1}$ u. A Abb.2). Unterstützend können visuelle Schemata helfen, dem Tierhalter den Status und das Ziel zu veranschaulichen ( Abb. 3 u. $\triangleright$ Abb. 4).

\section{Den Energiebedarf anschaulich vermitteln}

Zunächst muss der Tierhalter vermittelt bekommen, dass es keinen „allgemeingültigen Energiebedarf“ gibt, sondern sein Tier einen ganz individuellen Bedarf hat, abhängig von Körpergewicht und Aktivitätslevel, aber auch von z.B. Stoffwechselvorgängen und Thermoregulation. Ziel ist es also, den Energiebedarf seines eigenen Tieres individuell zu ermitteln und es entsprechend bedarfsgerecht zu ernähren. Der erste Schritt auf dem Weg zu diesem Ziel ist es, den ungefähren (durchschnittlichen) Energiebedarf abzuschätzen.
Der NRC, der nationale Forschungsrat der USA, legt seit vielen Jahren Werte für den täglichen Energiebedarf für Hunde und Katzen zugrunde, an denen sich die meisten Tierfuttermittelhersteller orientieren. Man kann jedoch davon ausgehen, dass diese im Jahr 2006 vom National Research Council (NRC) publizierten Energiebedarfsnormen für Hunde und Katzen unter heutigen Lebensbedingungen (abnehmende Aktivität) zu hoch angesetzt sind. Empfohlen werden daher im Kompendium als Basiswerte für:

- Hunde: $95 \mathrm{kcal} / \mathrm{kg} \mathrm{KGW}^{0,75}$ am Tag; dies entspricht beispielsweise für einen

- kleinen Hund mit 10 kg: ca. 530 kcal

- mittelgroßen Hund mit $20 \mathrm{~kg}$ : ca. $900 \mathrm{kcal}$

- großen Hund mit 30 kg: ca. $1220 \mathrm{kcal}$

- Katzen: 77,6 kcal/kg KGW ${ }^{0,71}$ am Tag; dies entspricht ca. $50 \mathrm{kcal} / \mathrm{kg} \mathrm{KGW}$, also bei einer $4 \mathrm{~kg}$ schweren, normalgewichtigen Katze ca. 200 kcal

Mars Petcare, Hersteller von marktführenden Marken wie Pedigree und Whiskas, hat als erstes Unternehmen auf diese Empfehlung reagiert und erste Rezepturen sowie die Fütterungsempfehlungen entsprechend angepasst. Bis Ende 2013 wird Mars Petcare diese Anpassung bei sämtlichen Produkten umsetzen.

Diese Zahlen können dem Tierhalter als Orientierung an die Hand gegeben werden, damit er die Ration seines Tieres entsprechend zusammenstellen kann. Aber es ist wichtig, dem Tierhalter zu erläutern, dass für die Bestimmung des individuellen Energiebedarfs die Körpermassekonstanz bei optimalem Ernährungszustand entscheidend ist.

Durch wöchentliches Wiegen des Tieres und die Prüfung der Körperkondition 
nach oben genannten Schemata - inklusive entsprechendem Protokollieren der Werte - wird deutlich, ob der zugrunde gelegte Energiebedarf und die daraus festgelegte Ration passend, zu gering oder zu hoch angesetzt sind. Sind sie nicht passend und nimmt das Tier deutlich ab oder zu, muss die Ration in einem zweiten Schritt korrigiert bzw. dem individuellen Energiebedarf angepasst werden.

\section{Energiegehalt verschiedener Nahrungen und Snacks verdeutlichen}

Entscheidend für die Beratung ist es, dem Tierhalter bewusst zu machen, welchen Effekt selbst „kleine Snacks“ auf die Energiebilanz haben und welche Konsequenzen dies für das Körpergewicht seines Tieres haben kann.

Zum Beispiel: Eine Scheibe Wurst liefert ca. $75 \mathrm{kcal}$, eine Scheibe Speck etwa 65 kcal. Gibt man einem mittelgroßen
Hund wie einem Cocker Spaniel nur eine Scheibe Wurst oder Speck pro Woche zusätzlich zur bedarfsdeckenden Tagesration, hat dieses kleine Plus das Potenzial, das Körpergewicht des Cocker Spaniels in nur einem Jahr um knapp ein Pfund zu steigern.

Ein anderes Beispiel: Hundekekse enthalten zwischen etwa 20 und 120 kcal pro Keks, Kauknochen aus Rinderhaut bis zu 700 kcal pro Stück und getrockneter Rinderpansen (40 g) ca. 160 kcal. Zahnpflegekauriegel zur täglichen Anwendung können weitere 50-70 kcal addieren, und größere Kauknochen zur wöchentlichen Anwendung enthalten bis zu 560 kcal. Gibt man einem Cocker Spaniel täglich einen mittelgroßen Hundekeks und einmal pro Woche ein Zahnpflegeprodukt, ohne dass die Hauptfutterration angepasst wird, so erhöht sich die wöchentliche Kalorienzufuhr um mehr als $300 \mathrm{kcal}$ und induziert damit u.U. eine jährliche Gewichtszunahme von mehr als $1,5 \mathrm{~kg}$.
Anhand solcher Beispiele - und mit Tabellen, in denen Snacks für Hunde und Katzen mit ihrem Energiegehalt vergleichend aufgeführt sind - kann dem Tierhalter eindrucksvoll vermittelt werden, dass Snacks und Leckerlis in der Gesamtration eine entscheidende Rolle einnehmen und neben den Hauptmahlzeiten in der Energiebilanz berücksichtigt werden müssen.

Wichtig ist darüber hinaus, dem Tierhalter den Unterschied in der Energiedichte zwischen Feucht- und Trockennahrung zu veranschaulichen. So enthält $100 \mathrm{~g}$ Trockennahrung im Schnitt genau so viele Kalorien wie $400 \mathrm{~g}$ Feuchtnahrung ( Abb.5). Es zeigt sich nämlich immer wieder, dass Hunde- und Katzenhalter die Rationen für ihr Tier „nach Augenmaß“ abmessen bzw. den Napf voll machen, unabhängig davon, ob Trockenoder Feuchtfutter verwendet wird. Sie sind gut beraten, sich die Rationen der einzelnen Futtermittel einmal exakt abzuwiegen und das Volumen der Ration

\section{Hund}

Ertasten Sie mit den Fingerspitzen (ohne Druck) gegen die

Haarwuchsrichtung leicht die Rippen?

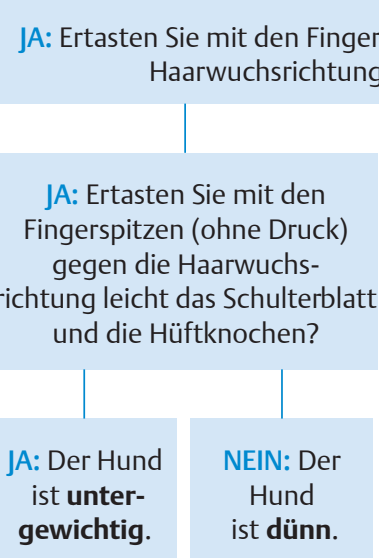

NEIN: Ertasten Sie mit den Fingerspitzen (mit leichtem Druck) gegen die Haarwuchsrichtung die Rippenkonturen?

NEIN: Der Hund ist schlank.

JA: Werden die Rippen von einer Fettschicht bedeckt?

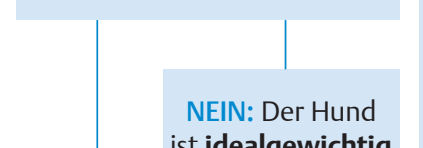
ist idealgewichtig.

J: Ertasten Sie, bei Streichen mit den Handflächen in Haarwuchsrichtung, zwischen Brustkorb und Hinterbeinen einen flacheren Bereich?

JA: Der Hund ist stämmig.
NEIN: Ertasten Sie, bei Streichen mit den Handflächen in Haarwuchsrichtung, die Hüfte (Einengung zwischen Ober- und Unterkörper)?

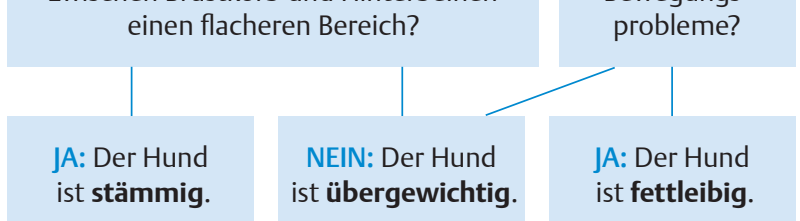

Abb. 1 WALTHAM ${ }^{\circledR}$ S.H.A.P.E ${ }^{T M}$ Guide für den Hund. 
mit den entsprechenden Kalorien in Verbindung zu bringen.

Im Rahmen von Forschungsarbeiten hat sich gezeigt, dass eine Kombination aus Feucht- und Trockennahrung, die sogenannte Mischfütterung, die Vorteile beider Futterarten optimal kombiniert und Wohlbefinden wie Gesundheit der Tiere bestmöglich unterstützt. Als Faustregel kann gelten, dass $2 / 3$ des Energiebedarfs über Feucht- und $1 / 3$ über Trockennahrung abgedeckt werden sollte.

\section{Ausreichend Bewegung, ein dehnbarer Begriff}

Bewegung ist ein wesentlicher Faktor für das vorbeugende Gewichtsmanagement. Um beim Tierhalter eine Verhaltensänderung zu erwirken, sollte er möglichst konkrete Tipps an die Hand bekommen.

Hundehalter sollten sich zumindest 2-mal am Tag Zeit für Auslauf und Spiel nehmen. Je nach Rasse, Alter, Größe und Gesundheitszustand des Tieres sollte der Tierarzt eindeutige Zeitangaben machen und erläutern, welche Art von Bewegung geeignet bzw. ungeeignet ist. Auch Hauskatzen sollten durch Jagd- und Suchspiele zur Bewegung animiert werden. Denn anders als bei Hundehaltern gehört die regelmäßige, gemeinsame Bewegung bei Katzenhaltern meist nicht zur Routine.

Die meisten Tierhalter überschätzen das Aktivitätsniveau ihrer Vierbeiner. Im Durchschnitt sind die Tiere weniger als eine Stunde pro Tag wirklich aktiv. Auch hier hilft ein Protokoll. Da der Alltag mit dem Tier meist regelmäßige, feste $\mathrm{Ab}$ läufe hat, reicht i.d.R. ein einziges Wochenprotokoll über die aktiv verbrachten Zeiten aus. Eine einfache Maßnahme, die dem Tierhalter das tatsächliche Maß an Aktivität sehr anschaulich verdeutlicht.

Vielfach können Situationen im Alltag für ein Mehr an Bewegung genutzt werden,
z.B. im Rahmen der Pflege oder Fütterung. Dazu zählen neben täglichen Spaziergängen mit Hunden bzw. dem Freilauf von Katzen u.a.:

- Interaktive Spiele: Ball-, Zerr-, Fangund Angelspiele.

- Spielerisches Füttern: Futterball, Verteilen/Verstecken von Trockennahrung, Futter als Leckerlis im Spiel geben.

- Kontakt mit Artgenossen: Verabredungen zum gemeinsamen Spaziergang, Hundetreffen, Hundesport, bei Katzen fördert die Gesellschaft/Haltung mit einer zweiten Katze nicht nur das Wohlbefinden, sondern auch die Aktivität.

- Gemeinsamer Sport: Nordic Walking, Inlineskaten, Fahrradfahren, Joggen.

- Zubehör für Katzen: Kratzbäume, Wippen, Kletterstangen, Podeste in der Wohnung. Wechselndes Spielzeug, gern auch Haushaltsgegenstände wie leere Toilettenrollen oder Joghurtbecher, um die Neugierde am Spiel zu erhalten.

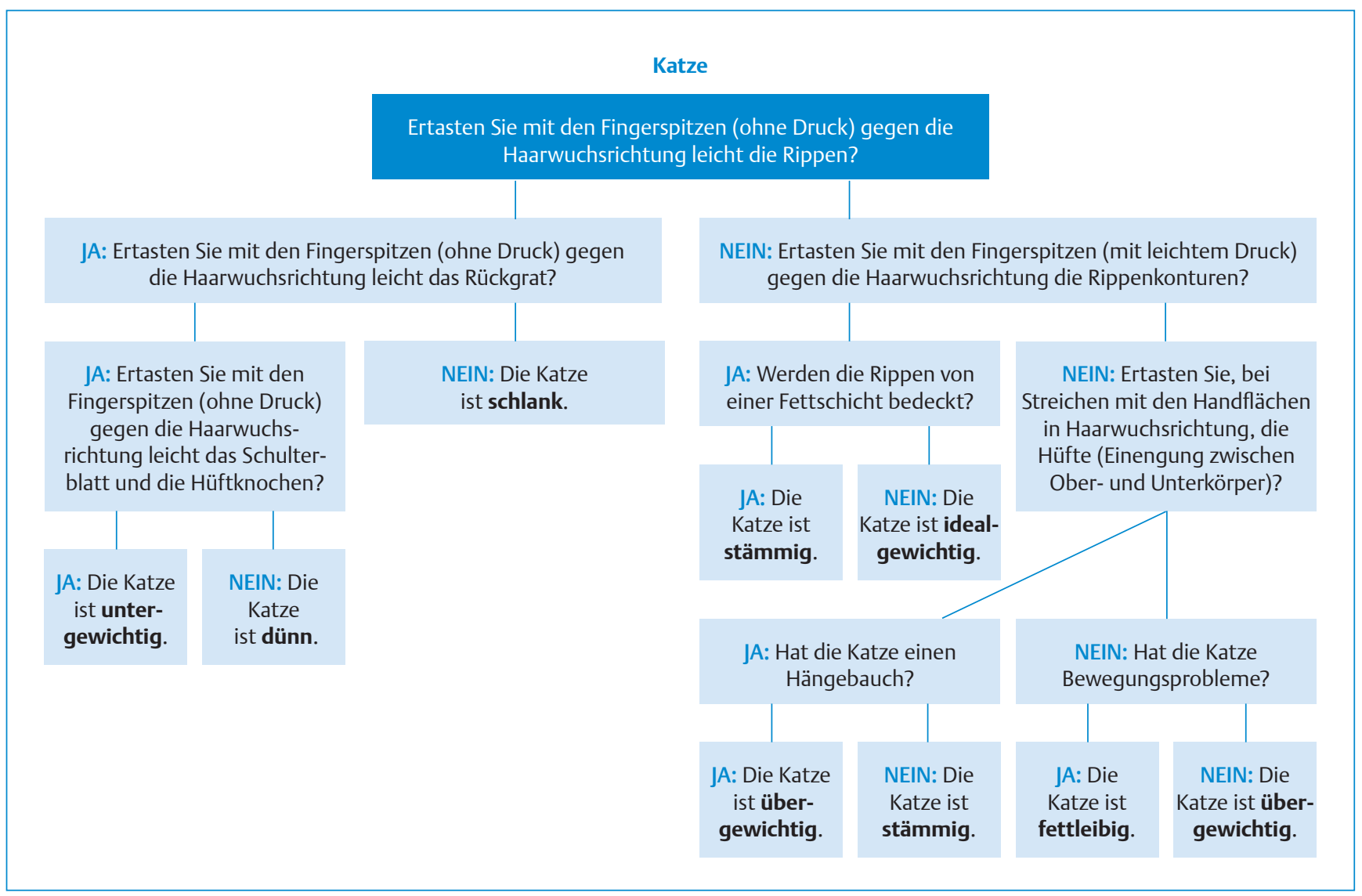

Abb. 2 WALTHAM ${ }^{\otimes}$ S.H.A.P.ETM Guide für die Katze. 
Letztlich aber - und auch dies sollte der Tierhalter wissen - gilt auch hier die individuelle Erfahrung mit dem eigenen Tier. Art und Maß der Bewegung müssen zu dem Tier passen, sowohl vom Temperament als auch vom Alter und Gesundheitszustand. Auch hier gilt individuell das klare Ziel: Zeigt das Gewichtsprotokoll, dass der Energiehaushalt des Vierbeiners nicht ausgeglichen ist, müssen Ernährung und/oder Bewegung entsprechend individuell angepasst werden.

\section{„Ja, aber ..." Ausflüchten be- gegnen, das Ziel klar machen}

Viele Tierhalter beteuern, dass sie sich strikt an einen Ernährungsplan halten, dass sie nichts nebenher füttern und dass das Tier ganz sicher ausreichend Bewegung hat. Als Erklärung für Übergewicht wird beispielsweise der besondere Stoffwechsel des Tieres angeführt, dass es schon immer leichtfuttrig war, dass es zu alt oder zu krank ist, um sich ausreichend zu bewegen, oder dass die Probleme erst nach der Kastration aufgetreten seien. Aber was auch immer tatsächlich dahinter steckt, das Problem muss angepackt werden, am besten ohne Schuldzuweisung. Ob es ein deutlich unterdurchschnittlicher Energiebedarf ist oder die täglich geteilte Tafel Schokolade ist dabei irrelevant, da sich beides voraussichtlich nach der Beratung nicht maßgeblich ändern wird. Tierärzte sollten daher freundlich Verständnis für all diese „Aus-
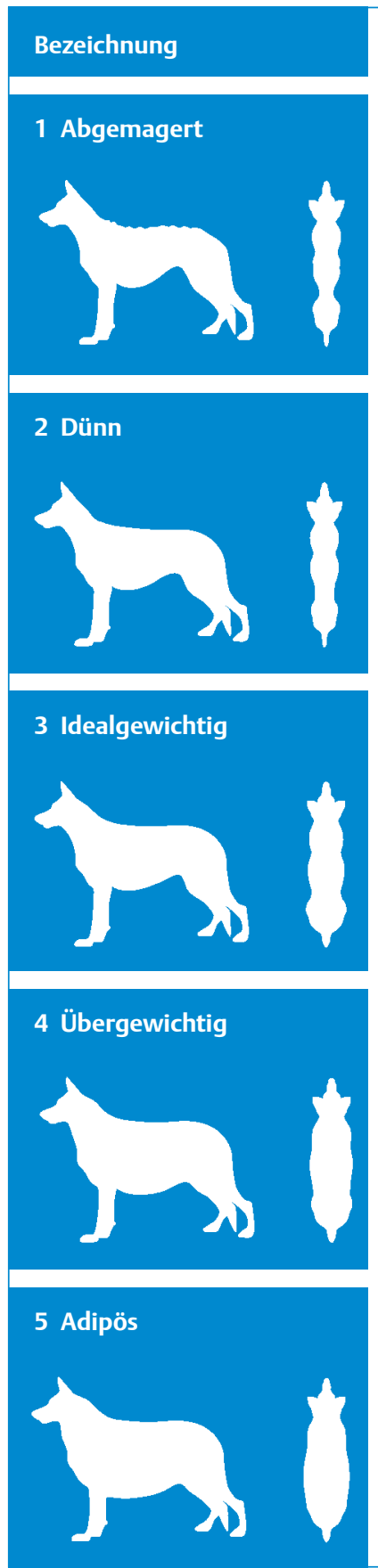

Abb. 3 Körperkonditionsindex Hund.

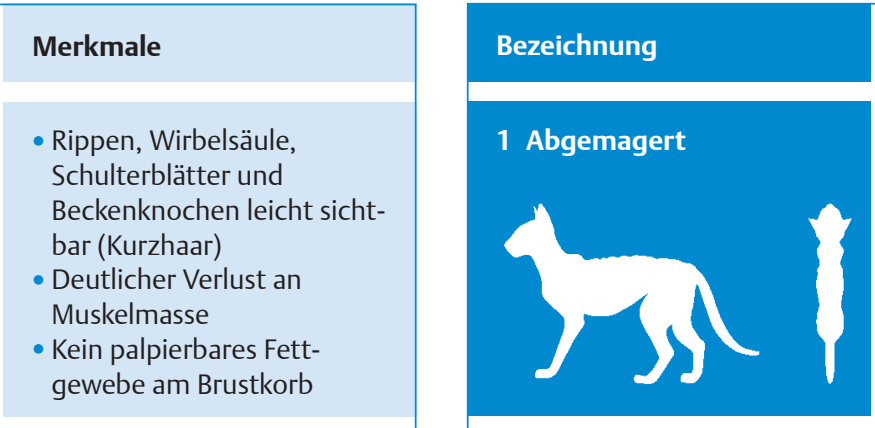

- Rippen, Wirbelsäule und Beckenknochen sichtbar

- Deutliche Taille

- Kein palpierbares Fettgewebe am Brustkorb

- Rippen und Wirbelsäule nicht sichtbar, aber leicht zu palpieren

- Deutliche Taille

- Dünne Fettgewebsschicht am Brustkorb palpierbar

- Rippen und Wirbelsäule nur schwer palpierbar

- Keine Taille erkennbar

- Deutliche Fettablagerungen entlang der Wirbelsäule und am Rutenansatz

- Massive Fettablagerungen am Brustkorb, entlang der Wirbelsäule und am Rutenansatz

- Deutliche Umfangs-

vermehrung des Abdomens

\section{Merkmale \\ - Rippen, Wirbelsäule, Schulterblätter und Beckenknochen leicht sicht- bar (Kurzhaarkatze) \\ - Deutlicher Verlust an \\ Muskelmasse \\ - Kein palpierbares Fett am Brustkorb}

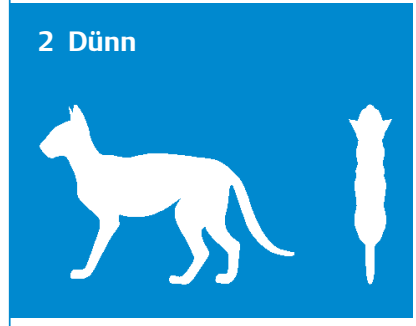

- Rippen, Wirbelsäule, Schulterblätter und Beckenknochen sichtbar

- Deutliche Taille

- Geringe Menge Bauchfett palpierbar

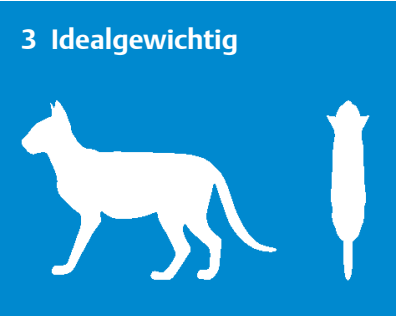

- Rippen und Wirbelsäule nicht sichtbar, aber leicht zu palpieren

- Deutliche Taille

- Geringe Menge Bauchfett palpierbar

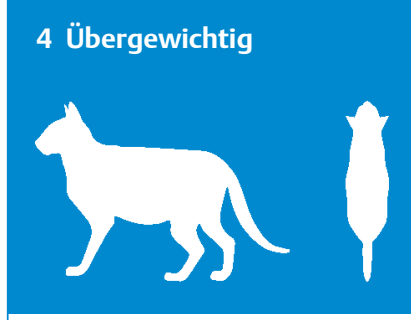

- Rippen und Wirbelsäule nur schwer palpierbar

- Keine Taille erkennbar

- Deutliche Erweiterung des Abdomens (ventrales Bauchfettdepot)

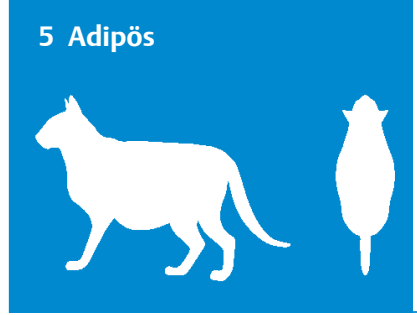

- Massive Fettablagerungen über dem Brustkorb, an der Wirbelsäule und am Bauch

- Massive Erweiterung des Abdomens („Hängebauch“)

Abb. 4 Körperkonditionsindex Katze. 


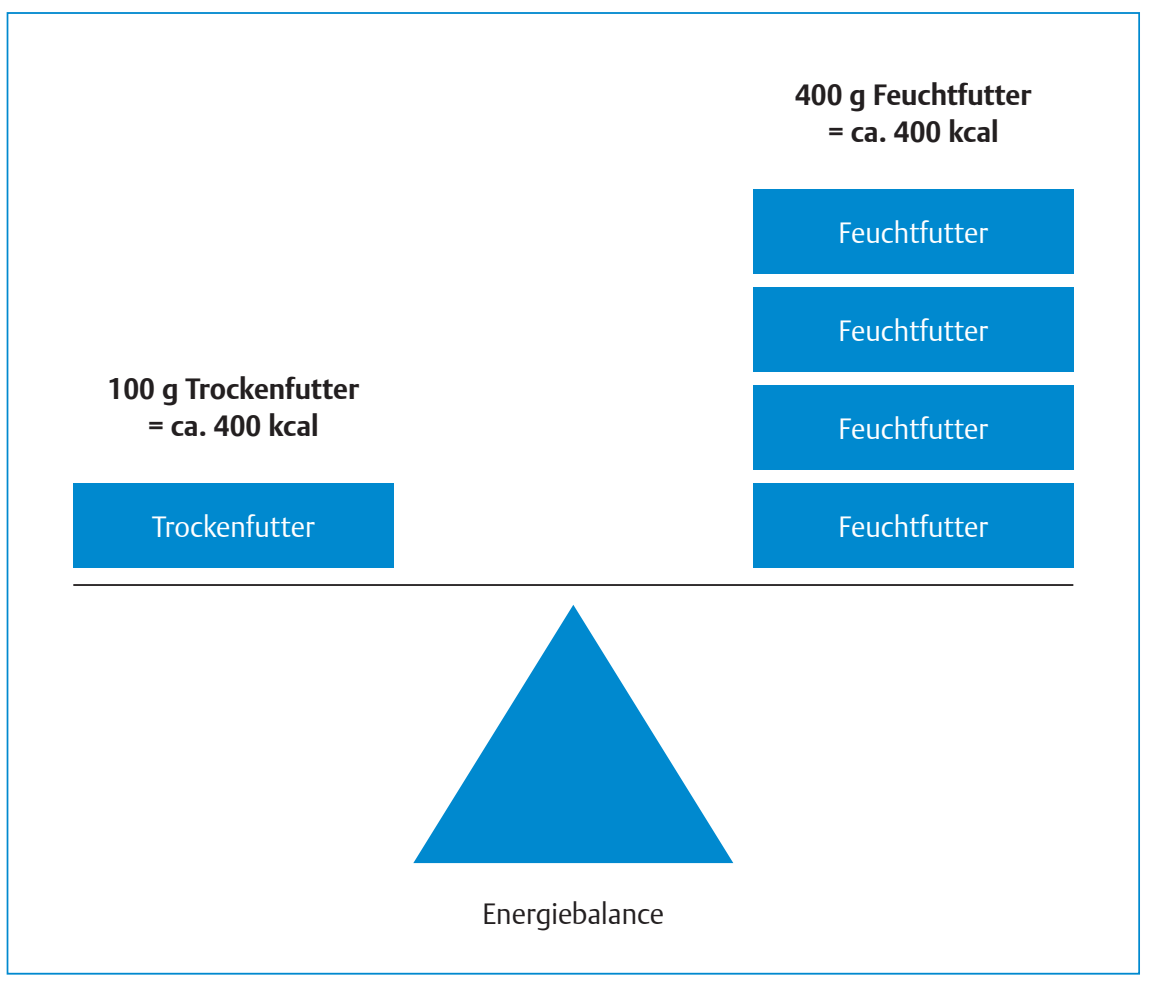

Abb. 5 Vergleich der Kaloriendichte von Trocken- und Feuchtfutter. flüchte“ zeigen (auch, damit sie die relevanten Informationen vom Tierhalter tatsächlich erhalten) und sich darauf konzentrieren, das Ziel zu verdeutlichen:

Hund oder Katze sollen gesund sein und daher auch eine gute Körperkondition aufweisen, damit keine Sekundärerkrankungen drohen und Wohlbefinden sowie Lebensdauer des Tieres beeinträchtigen. Ein allgemeingültiges Patentrezept dafür gibt es nicht, es geht um den individuellen Patienten - und für dieses Tier muss ein individuell passender Weg zum Erhalt des Idealgewichtes gefunden werden.

Ganz gleich, ob kastriert oder gemütlich, ob alt oder ein Freund von leckerem Futter: Den richtigen Weg für sein Tier kann und muss der Tierhalter dauerhaft finden, indem er sich folgende Tipps zu Herzen nimmt:

1. Energiebedarf ermitteln: Beginnend mit einer ersten Berechnung auf Basis des Normalgewichtes als Orientie- 
rung. Anschließend (kontinuierlich) anpassen an individuelle Gegebenheiten und den Verlauf der Gewichtsentwicklung.

2. Gewicht und Körperkondition regelmäßig kontrollieren.

3. Kaloriengehalt verschiedener Futtermittel und Snacks sowie deren Konsequenz auf die Energiebilanz stets deutlich machen.

4. Ernährungsprotokoll erstellen, um objektiv festzuhalten, wie viele Kalorien verfüttert werden.

5. Bewegung fördern, ggf. auch protokollieren.

6. Zusammenfassend oben stehende Faktoren in Verbindung bringen, ggf. anpassen - so lange bis Ernährung und Bewegung ein konstantes, gesundes Körpergewicht ergeben.

7. Dauerhaft mindestens $1 \times$ monatlich Überprüfung des Körpergewichts. Bei bedenklichen Veränderungen mit Punkt 1 bis 6 neu beginnen.

\section{Online zu finden unter}

http://dx.doi.org/10.1055/s-0032-1331074

\section{Literatur}

Literatur bei der Verfasserin.

\section{Dr. Britta Dobenecker}

FTA, Dipl. ECVCN

Veterinärwissenschaftliches Department

Lehrstuhl für Tierernährung und Diätetik

Tierärztliche Fakultät Ludwig-Maximilians-

Universität München

Schönleutnerstr. 8, 85764 Oberschleißheim 